\title{
On Idempotent Measures of Small Norm
}

\author{
Jayden Mudge
}

VICTORIA UNIVERSITY OF WELLINGTON

Te Whare Wānanga o te Ūpoko o te Ika a Māui

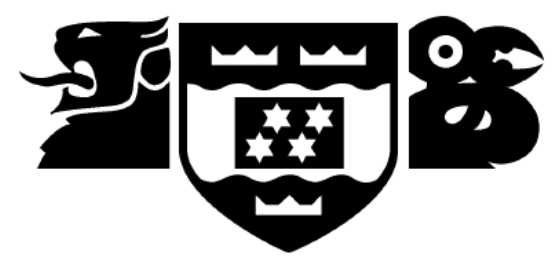

School of Mathematics and Statistics

Te Kura Mātai Tatauranga

\begin{abstract}
A thesis
submitted to the Victoria University of Wellington in fulfilment of the requirements for the degree of

Master of Science

in Mathematics.
\end{abstract}

Victoria University of Wellington

2016 



\begin{abstract}
In this Master's Thesis, we set up the groundwork for [8], a paper co-written by the author and Hung Pham.

We summarise the Fourier and Fourier-Stieltjes algebras on both abelian and general locally compact groups. Let $\Gamma$ be a locally compact group. We answer two questions left open in [11] and [13]:
\end{abstract}

1. When $\Gamma$ is abelian, we prove that if $\chi_{S} \in B(\Gamma)$ is an idempotent with norm $1<$ $\left\|\chi_{S}\right\|<\frac{4}{3}$, then $S$ is the union of two cosets of an open subgroup of $\Gamma$.

2. For general $\Gamma$, we prove that if $\chi_{S} \in M_{c b} A(\Gamma)$ is an idempotent with norm $\left\|\chi_{S}\right\|_{c b}<$ $\frac{1+\sqrt{2}}{2}$, then $S$ is an open coset in $\Gamma$. 



\section{Acknowledgements}

I would like to thank my supervisor Hung Pham, for the countless hours he dedicated to helping, Victoria University Scholarship office for funding me with the Master's (by thesis) scholarship, and all my friends for the support and overwhelming number of hugs.

In particular, I would like to thank my fellow students Finnian Gray, Ben Deeble, Susan Jowett, Jasmine Hall and Matt Grice for their wonderful companionship throughout the year.

Finally, I would like to thank my parents Neil and Julie Mudge for helping support me through these trying times. 



\section{Contents}

1 Preliminaries 1

1.1 Locally compact groups $\ldots \ldots \ldots \ldots$. . . . . . . . . . . . 1

1.2 The dual of a locally compact abelian group $\ldots \ldots \ldots 2$

1.3 Annihilators of locally compact abelian groups $\ldots \ldots$. . . . . . . . . 4

\begin{tabular}{|lll}
\hline 2 & The Fourier-Stieltjes and Fourier algebras & 7
\end{tabular}

2.1 Complex measures $\ldots \ldots \ldots \ldots \ldots \ldots$

2.2 The Fourier-Stieltjes algebra $B(\Gamma) \ldots \ldots \ldots \ldots$

2.3 The Haar Measure . . . . . . . . . . . . . . . . . . . . . . 11

$2.4 \quad$ The integrable function space $L^{1}(G) \ldots \ldots \ldots \ldots \ldots$

2.5 The Fourier algebra $A(\Gamma) \ldots \ldots \ldots \ldots \ldots$

\begin{tabular}{|ll|}
\hline & Idempotents of small norm on LCA groups \\
\hline
\end{tabular}

3.1 Idempotent measures $\ldots \ldots \ldots \ldots \ldots \ldots$. . . . . . . . . . . 21

3.2 Idempotents of norm less than $\frac{4}{3} \ldots \ldots \ldots \ldots \ldots$

4 Completely bounded Schur multipliers 29

$4.1 \quad$ Multipliers, and completely bounded multipliers . . . . . . . . . . . . . . 29

4.2 Idempotent completely bounded multipliers . . . . . . . . . . . . 31 


\section{Chapter 1}

\section{Preliminaries}

\subsection{Locally compact groups}

Within this and the following chapter, we summarise information from [2], [5] and [9]. These texts provide greater insight into the topics of topological groups, measure theory and harmonic analysis, however we will only outline the theorems that are of particular use to us for chapters 3 and 4 .

Definition 1.1.1. A Hausdorff topological space $X$ is called locally compact if every point $x \in X$ has an open neighbourhood whose closure is compact.

A locally compact group is a group $G$ equipped with a locally compact topological structure, such that the operations $(x, y) \mapsto x y$ and $x \mapsto x^{-1}$ are continuous with respect to the topologies of $G \times G \rightarrow G$ and $G \rightarrow G$ respectively.

For any subset $A \subseteq G$, and any $x \in G$, we will use the conventions

$$
A x=\{a x: a \in A\} \quad x A=\{x a: a \in A\} \quad A^{-1}=\left\{a^{-1}: a \in A\right\}
$$

For some $B \subseteq G$, we will have $A B=\{a b: a \in A, b \in B\}$.

Proposition 1.1.2. For any open subset $U \subseteq G$, then for any $x \in G$ and $A \subseteq G$, we have $x U, U x, U^{-1}, A U$ and $U A$ open in $G$.

Unless stated otherwise, we shall use multiplicative notation $x y$ for a general group, and call the identity element $e$. If we are assuming the group is abelian, then we shall use additive notation $x+y$, and call the identity element 0 . 


\subsection{The dual of a locally compact abelian group}

Definition 1.2.1. Let $G$ be a locally compact abelian group. We define the dual group $\widehat{G}$ of $G$ to be

$$
\widehat{G}=\{\gamma: G \rightarrow \mathbb{T}: \gamma \text { is a continuous homomorphism }\}
$$

For $\gamma_{1}, \gamma_{2} \in \widehat{G}$, we define $\left(\gamma_{1}+\gamma_{2}\right)(x)=\gamma_{1}(x) \gamma_{2}(x)$, and have the constant function $\widehat{0}(x)=1$ as the identity in $\widehat{G}$. For any $\gamma \in \widehat{G}$, we have $(-\gamma)(x)=\gamma(x)^{-1}=\overline{\gamma(x)}$.

It is convention to write $(x, \gamma)$ in place of $\gamma(x)$, in which case we have $\left(x_{1}+x_{2}, \gamma\right)=$ $\left(x_{1}, \gamma\right)\left(x_{2}, \gamma\right)$, and $\left(x, \gamma_{1}+\gamma_{2}\right)=\left(x, \gamma_{1}\right)\left(x, \gamma_{2}\right)$.

The topology on $\widehat{G}$ is the topology of compact convergence. In particular, a neighbourhood base for some point $\gamma_{0} \in \widehat{G}$ consists of all the sets of the form

$$
U_{\varepsilon, K}=\left\{\gamma \in \widehat{G}:\left|1-\left(x, \gamma-\gamma_{0}\right)\right|<\varepsilon, \forall x \in K\right\}
$$

for $\varepsilon>0$, and $K$ a compact subset of $G$.

Throughout this text, we shall denote $\widehat{G}$ as $\Gamma$.

Proposition 1.2.2. The dual $\Gamma$ of a locally compact abelian group $G$ is a topological abelian group.

Proof. It is obvious that $\Gamma$ is an abelian group, so we must check that the operations of (a) $+: \Gamma \times \Gamma \rightarrow \Gamma$ and (b) $-: \Gamma \rightarrow \Gamma$ are continuous.

a) Take $\gamma_{1}, \gamma_{2} \in \Gamma$. For compact, non-empty $K \subset G$, and $\varepsilon>0$, the set $U_{2 \varepsilon, K}$, and its translates, are open in $\Gamma$, thus $\left(\gamma_{1}+U_{\varepsilon, K}\right) \times\left(\gamma_{2}+U_{\varepsilon, K}\right)$ is an open neighbourhood of $\left\langle\gamma_{1}, \gamma_{2}\right\rangle \in \Gamma \times \Gamma$.

Via the triangle inequality, if $\eta_{1} \in \gamma_{1}+U_{\varepsilon, K}$ and $\eta_{2} \in \gamma_{2}+U_{\varepsilon, K}$, for all $x \in K$, we have

$$
\begin{aligned}
\left|\left(x, \gamma_{1}+\gamma_{2}\right)-\left(x, \eta_{1}+\eta_{2}\right)\right| & =\left|\left(x, \gamma_{1}\right)\left(x, \gamma_{2}\right)-\left(x, \eta_{1}\right)\left(x, \eta_{2}\right)\right| \\
& =\left|\left(x, \gamma_{2}\right)\left[\left(x, \gamma_{1}\right)-\left(x, \eta_{1}\right)\right]+\left(x, \eta_{1}\right)\left[\left(x, \gamma_{2}\right)-\left(x, \eta_{2}\right)\right]\right| \\
& \leq\left|\left(x, \gamma_{1}\right)-\left(x, \eta_{1}\right)\right|+\left|\left(x, \gamma_{2}\right)-\left(x, \eta_{2}\right)\right| \\
& <\varepsilon+\varepsilon=2 \varepsilon
\end{aligned}
$$

Hence $\left\langle\eta_{1}, \eta_{2}\right\rangle$ must be in $\left(\gamma_{1}+U_{\varepsilon, K}\right) \times\left(\gamma_{2}+U_{\varepsilon, K}\right)$, proving + is continuous.

b) Again, for any $\varepsilon>0$ and compact, non-empty $K \subseteq G$, let us define $\gamma+U_{\varepsilon, K}$ to be an open neighbourhood of $\gamma$. For any $\eta \in \gamma+U_{\varepsilon, K}$, and for all $x \in K$, we have 


$$
\begin{aligned}
|(x,-\gamma)-(x,-\eta)| & =|\overline{(x, \gamma)}-\overline{(x, \eta)}| \\
& =|\overline{(x, \gamma)-(x, \eta)}| \\
& =|(x, \gamma)-(x, \eta)|<\varepsilon
\end{aligned}
$$

Hence $-\eta \in-\left(\gamma+U_{\varepsilon, K}\right)$, so - must also be continuous.

Theorem 1.2.3. The dual group $\Gamma$ of a locally compact abelian group $G$ is a locally compact abelian group.

As we know $\Gamma$ is an abelian topological group, all that remains to be seen is that the topological structure is locally compact. However, this requires additional machinery we do not wish to cover it in this text, so the proof shall be excluded. It can be found in [9].

Proposition 1.2.4. The dual group of a compact group is discrete.

Proof. Assume $G$ is compact, and take $\gamma_{0} \in \Gamma$ to be arbitrary.

Then all sets of the form $U_{\varepsilon}=\left\{\gamma:\left|1-\left(x, \gamma_{0}^{-1} \gamma\right)\right|<\varepsilon, x \in G\right\}$ are the open neighbourhoods of $\gamma_{0}$, for any $\varepsilon>0$. If we fix $\varepsilon=\sqrt{2}$, then we must have the set

$$
U_{\sqrt{2}}=\left\{\gamma:\left|1-\left(x, \gamma_{0}^{-1} \gamma\right)\right|<\sqrt{2}, x \in G\right\}
$$

as an open set in $\Gamma$. Surely $\gamma_{0}$ is in $U_{\sqrt{2}}$, but assume some $\gamma_{1} \neq \gamma_{0}$ is also in $U_{\sqrt{2}}$. Then $\left|1-\left(x, \gamma_{0}^{-1} \gamma_{1}\right)\right|<\sqrt{2}$ for all $x \in G$.

Take some $x_{0}$ such that $z:=\left(x_{0}, \gamma_{0}^{-1} \gamma_{1}\right) \in \mathbb{T}$ satisfies $z \neq 1$. For some $n \in \mathbb{N}$, we must have $\operatorname{Re}\left(z^{n}\right)<0$. Hence $\left|1-z^{n}\right| \geq \sqrt{2}$, and more directly, $\left|1-\left(x_{0}^{n}, \gamma_{0}^{-1} \gamma_{1}\right)\right| \geq \sqrt{2}$. This contradicts the definition of $U_{\sqrt{2}}$, hence $\gamma_{1} \notin U_{\sqrt{2}}$, and indeed $U_{\sqrt{2}}=\left\{\gamma_{0}\right\}$.

Theorem 1.2.5 (Pontryagin duality theorem). The dual group of $\Gamma$ is $G$.

The proof of this result can be found in [9].

Corollary 1.2.6. The dual group of a discrete group is compact. 
Corollary 1.2.7. Sets of the form

$$
U_{\varepsilon, K}=\{\gamma \in \Gamma:|1-(x, \gamma)|<\varepsilon, \forall x \in K\}
$$

for compact $K \subseteq G, \varepsilon>0$, and their translates, form an open neighbourhood base for $G$.

Proof. This is simply the topology of compact convergence on $\Gamma$, hence is the topology of $G$ by the Pontryagin duality theorem.

The above statement is proved as Proposition 1.2.6. in [9], without reference to the Pontryagin duality theorem, as it is a key part of the theorem's proof. As it is a natural consequence of the theorem, we are happy to accept it within this paper.

Example 1.2.8. Let $\mathbb{Z}$ be the integers equipped with addition and the discrete topology, $\mathbb{T}$ be the unit circle with multiplication and the standard topology, and $\mathbb{R}$ be the real numbers with addition and the standard topology. Then we have

1. $\widehat{\mathbb{T}}=\mathbb{Z}$, via the map $\left(n, e^{i \theta}\right)=e^{i n \theta}$ for each $n \in \mathbb{Z}, e^{i \theta} \in \mathbb{T}$.

2. $\widehat{\mathbb{Z}}=\mathbb{T}$, via the map $\left(e^{i \theta}, n\right)=e^{i n \theta}$ for each $e^{i \theta} \in \mathbb{T}, n \in \mathbb{Z}$.

3. $\widehat{\mathbb{R}}=\mathbb{R}$, via the map $(x, y)=e^{2 \pi i x y}$ for each $x, y \in \mathbb{R}$.

4. $\widehat{\mathbb{Z}_{n}}=\mathbb{Z}_{n}$, via the map $(a, b)=e^{2 \pi i a b / n}$ for each $a, b \in \mathbb{Z}_{n}$

It also follows that every finite abelian group is self dual.

\subsection{Annihilators of locally compact abelian groups}

Definition 1.3.1. Let $H$ be a subgroup of an locally compact abelian group $G$. We call the set

$$
\Lambda=\{\gamma \in \Gamma:(x, \gamma)=1, \forall x \in H\}
$$

the annihilator of $H$.

Proposition 1.3.2. If $\Lambda$ is the annihilator of $H$, then $\Lambda$ is a subgroup of $\Gamma$. Furthermore, the annihilator of $\Lambda$ is $H$.

Proof. Certainly $0 \in \Lambda$, for $(x, 0)=1$. For any $\gamma \in \Lambda,(x, \gamma)=1=(x,-\gamma)$ for all $x \in H$, as 1 is real valued, and finally if $\gamma_{1}, \gamma_{2} \in \Lambda$, then $\left(x, \gamma_{1}+\gamma_{2}\right)=\left(x, \gamma_{1}\right)\left(x, \gamma_{2}\right)=1$.

Denote by $\Lambda^{\perp}$ the annihilator of $\Lambda$ in $G$. Clearly $H \subseteq \Lambda^{\perp}$, as $(x, \gamma)=1$ for all $x \in H, \gamma \in \Lambda$. If $H=G$, then $\Lambda=\{0\}$, and the result is obvious. Instead consider that $H$ is a strict subgroup of $G$, and there some $x_{0} \in \Lambda^{\perp}$ that is not in $H$. Then $G / H$ is a nontrivial 
group of $G$, and there exists some dual member $\phi: G / H \rightarrow \mathbb{T}$ that is not the identity map. The map $\gamma: x \mapsto(x+H, \phi)$ is a continuous homomorphism, hence is in $\Gamma$, and satisfies $(x, \gamma)=1$ whenever $x \in H$; this must mean that $\gamma$ is in fact a member of $\Lambda$. Ergo $\left(x_{0}, \gamma\right)=\left(x_{0}+H, \phi\right) \neq 1$, and $x_{0}$ cannot be in $\Lambda^{\perp}$, thus completing the proof.

Not only are $H$ and $\Lambda$ mutually annihilators for each other, but they are deeply related to the quotient group structure within $G$ and $\Gamma$.

Theorem 1.3.3. Let $G$ be a locally compact abelian group, with subgroup $H$, let and $\Lambda$ be the annihilator of $H$ in $\Gamma$. Then $\Lambda$ is isomorphic to $\widehat{G / H}$, and $\Gamma / \Lambda$ is isomorphic to $\widehat{H}$, both in a homeomorphic way.

Proof. For any $x \in G$, there is a natural, open and continuous homomorphism $h(x)=x+H$ onto $G / H$. For any $\phi \in \widehat{G / H}$, we can create a map $\imath: \widehat{G / H} \rightarrow \Lambda$ defined by $\imath(\phi):=\phi \circ h$. Then $\imath$ is injective, as if $\imath\left(\phi_{1}\right)=\imath\left(\phi_{2}\right)$, then $\phi_{1} \circ h=\phi_{2} \circ h$. This means $\phi_{1} \circ h(x)=\phi_{2} \circ h(x)$ for all $x \in G$, and $\phi_{1}(x+H)=\phi_{2}(x+H)$ for all $x+H \in G / H$, so we get $\phi_{1}=\phi_{2}$.

The map $\imath$ is also surjective, as for any $\gamma \in \Lambda$, we can consider $\gamma \circ h^{-1}$ as a member of $\widehat{G / H}$. This is because, for any coset $x+H \in G / H$, the inverse $h^{-1}(x+H)=\{x+a: a \in$ $H\}$, and as $\gamma$ annihilates $H$, we have $\gamma(x+a)=\gamma(x)$ for all $a \in H$, hence the formula $\gamma \circ h^{-1}$ is well-defined. It can easily be shown that $\gamma \circ h^{-1}$ is a continuous homomorphism on $G / H$, and as $\imath\left(\gamma \circ h^{-1}\right)=\gamma, \Lambda$ and $\widehat{G / H}$ are bijective.

We also have that $l$ is an homomorphism, as

$$
\left(x, \imath\left(\phi_{1}+\phi_{2}\right)\right)=\left(h(x), \phi_{1}+\phi_{2}\right)=\left(h(x), \phi_{1}\right)\left(h(x), \phi_{2}\right)=\left(x, \imath\left(\phi_{1}\right)+\imath\left(\phi_{2}\right)\right) \quad(x \in G)
$$

For any compact set $K_{1} \subseteq G$, the set $h\left(K_{1}\right)$ is compact in $G / H$, and for any compact $K_{2} \subseteq$ $G / H$, there is some compact $K_{1} \subseteq G$ such that $h\left(K_{1}\right)=K_{2}$, as $h$ is open and continuous. By 1.2.7, the sets

$$
U_{\varepsilon, K_{1}}=\left\{\gamma \in \Lambda:|1-(x, \gamma)|<\varepsilon, \forall x \in K_{1}\right\}
$$

form an open neighbourhood base of $\Lambda$, and $\imath$ maps any $U_{\varepsilon, K_{1}}$ onto the open set $U_{\varepsilon, K_{2}}$, where

$$
U_{\varepsilon, K_{2}}=\left\{\phi \in \widehat{G / H}:|1-(w, \phi)|<\varepsilon, \forall w \in K_{2}\right\}
$$

Furthermore, for any open set of the form $U_{\varepsilon, K_{2}}$, there is some open $U_{\varepsilon, K_{1}}$ such that $\imath\left(U_{\varepsilon, K_{1}}\right)=U_{\varepsilon, K_{2}}$, hence $\imath$ is a homeomorphism, thus it is a homeomorphic isomorphism from $\widehat{G / H}$ to $\Lambda$.

As $H$ and $\Lambda$ are mutual annihilators, $\widehat{\Gamma / \Lambda}$ is isomorphic to $H$, and so by the Pontryagin duality theorem, $\Gamma / \Lambda$ is isomorphic to $\widehat{H}$, and the theorem is proved.

Corollary 1.3.4. The annihilator of an open subgroup is compact, and the annihilator of a compact subgroup is open.

Proof. Let $H$ be an open subgroup of $G$, with annihilator $\Lambda$. Then $G / H$ is discrete, and its dual $\Lambda$ is compact. The rest follows from mutual annihilation. 


\section{Chapter 2}

\section{The Fourier-Stieltjes and Fourier algebras}

\subsection{Complex measures}

Definition 2.1.1. Let $(X, \mathscr{M})$ be a measurable space. A complex measure $\mu$ on $(X, \mathscr{M})$ is a function from $\mathscr{M}$ to $\mathbb{C}$ that satisfies

$$
\mu(\emptyset)=0 \quad \text { and } \quad \mu\left(\bigcup_{n=1}^{\infty} A_{n}\right)=\sum_{n=1}^{\infty} \mu\left(A_{n}\right)
$$

for any disjoint sequence $A_{n}$ in $\mathscr{M}$.

Any complex measure can be written in the form $\mu=\mu_{r}+i \mu_{i}$, where $\mu_{r}, \mu_{i}$ are finite signed measures on $(X, \mathscr{M})$, and via the Jordan Decomposition theorem,

$$
\mu=\mu_{1}-\mu_{2}+i \mu_{3}-i \mu_{4}
$$

for $\mu_{n}$ finite positive measures on $(X, \mathscr{M})$. The total variation of a measure $|\mu|$ of a complex measure $\mu$ is defined to be the positive measure $|\mu|(A)=\sup \sum_{j=1}^{n}\left|\mu\left(A_{j}\right)\right|$, this supremum taken over all finite partitions of $A$ into $\mathscr{M}$-measurable sets.

The total variation norm of a complex measure $\mu$ is defined to be $\|\mu\|=|\mu|(X)$.

It can also be seen that $|\mu|(E) \leq \mu_{1}(E)+\mu_{2}(E)+\mu_{3}(E)+\mu_{4}(E)$ for measurable $E \subseteq X$.

Proposition 2.1.2. Let $M(X, \mathscr{M})$ denote the space of all complex measures on $(X, \mathscr{M})$. Then $M(X, \mathscr{M})$ is a complex vector space, and the total variation norm $\|\mu\|$ is indeed a norm on this space.

$M(X, \mathscr{M})$, equipped with the total variation norm, forms a Banach space. 
Definition 2.1.3. The Borel $\sigma$-algebra of $X$ is the $\sigma$-algebra generated by the open subsets of $X$. A measure on $X$ is called a Borel measure if it is defined on the Borel measurable sets, and a function $f$ is called a Borel function if it is Borel measurable.

If $\mu$ is a Borel measure on $X$, then we say $\mu$ is

i. Outer regular if for each open set $U, \mu(U)=\sup \{\mu(K): K \subseteq U$, for compact $K\}$

ii. Inner regular if for each measurable set $A, \mu(A)=\inf \{\mu(U): A \subseteq U$, for open $U\}$

iii. Regular if it is both outer and inner regular, and each compact $K$ satisfies $\mu(K)<\infty$.

In the case that our topological space $G$ is a locally compact group, we will write $M(G)$ to denote the space of all regular Borel measures on $G$.

Proposition 2.1.4. Let $\mu \in M(G)$. For any bounded $f$ on $G$, the inequality $\left|\int f d \mu\right| \leq$ $\|f\|_{\infty}\|\mu\|$ holds.

Proof. First, let $f$ be a simple function. Then there exists values $a_{1}, \cdots a_{k}$ and sets $A_{1}, \cdots, A_{k}$ such that

$$
\left|\int f d \mu\right|=\left|\sum_{j=1}^{k} a_{j} \mu\left(A_{j}\right)\right| \leq \sum_{j=1}^{k}\left|a_{j}\right|\left|\mu\left(A_{j}\right)\right| \leq\|f\|_{\infty}\|\mu\|
$$

It is well known any Borel function $f$ can be expressed as the uniform limit of a increasing sequence of simple functions $\left(f_{n}\right)$ on $G$, and so

$$
\begin{aligned}
\left|\int\left(f-f_{n}\right) d \mu\right| & =\left|\int\left(f-f_{n}\right) \mu_{1}-\int\left(f-f_{n}\right) \mu_{2}+i \int\left(f-f_{n}\right) \mu_{3}-i \int\left(f-f_{n}\right) \mu_{4}\right| \\
& \leq \sum_{j=1}^{4}\left|\int\left(f-f_{n}\right) d \mu_{j}\right| \\
& \leq \sum_{j=1}^{4} \int\left|f-f_{n}\right| d \mu_{j} \leq\left\|f-f_{n}\right\|_{\infty} \sum_{j=1}^{4}\left\|\mu_{j}\right\|
\end{aligned}
$$

and indeed we have $\int f_{n} d \mu \rightarrow \int f d \mu$ as $n \rightarrow \infty$, hence $\left|\int f_{n} d \mu\right| \rightarrow\left|\int f d \mu\right|$ also.

As $\left|\int f_{n} d \mu\right| \leq\left\|f_{n}\right\|_{\infty}\|\mu\|$ for all simple functions, it follows that our inequality holds for all Borel functions of $G$.

Definition 2.1.5. Let $G$ be a locally compact group. For $\mu, v \in M(G)$, we can define the convolution $\mu * v$ to be a measure

$$
(\mu * v)(E)=(\mu \times v)(D), \quad \text { where } D=\{(x, y) \in G \times G: x y \in E\}
$$

Convolution of measures is associative, and is commutative if and only if $G$ is abelian. Furthermore, we have $\|\mu * v\| \leq\|\mu\|\|v\|$, so in fact $M(G)$ is a Banach Algebra, when equipped with convolution. 


\subsection{The Fourier-Stieltjes algebra $B(\Gamma)$}

Definition 2.2.1. Let $G$ be a locally compact abelian group. For $\mu \in M(G)$, we define the Fourier-Stieltjes transform $\widehat{\mu}$ to be the function

$$
\widehat{\mu}: \gamma \mapsto \int_{G}(-x, \gamma) d \mu(x) \quad(\forall \gamma \in \Gamma)
$$

Proposition 2.2.2. The map ${ }^{\wedge}: \mu \mapsto \widehat{\mu}$ is a algebra homomorphism from $M(G)$ into $L^{\infty}(\Gamma)$.

Proof. We can see the Fourier-Stieltjes transform preserves multiplication, as

$$
\begin{aligned}
\widehat{\mu * v}(\xi) & =\int(-z, \xi) d(\mu * v)(z) \\
& =\iint(-x-y, \xi) d \mu(x) d v(y) \quad(x+y=z) \\
& =\int(-x, \xi) d \mu(x) \int(-y, \xi) d v(y) \\
& =\widehat{\mu} \widehat{v}
\end{aligned}
$$

Furthermore, $\widehat{\mu}$ is bounded, as $|\widehat{\mu}(\gamma)|=\left|\int(x, \gamma) d \mu(x)\right| \leq\|\mu\|$

The image of $M(G)$ under the Fourier-Stieltjes Transform is called the Fourier-Stieltjes algebra, and is denoted $B(\Gamma)$. We define the norm of $B(\Gamma)$ to simply be $\|\widehat{\mu}\|_{B(\Gamma)}:=$ $\|\mu\|_{M(G)}$.

Theorem 2.2.3. For $\mu, v \in M(G)$, if $\widehat{\mu}=\widehat{v}$, then $\mu=v$.

This is called the Fourier Uniqueness theorem. The proof is very long and involved, but it can be found in [9], on pages 17-30. As we are not proving this result in this paper, we shall try not to rely too heavily on it. However, it is essential for Chapter 3 .

Definition 2.2.4. A function $\phi: G \rightarrow \mathbb{C}$ is called positive definite if for all $c_{1}, \cdots, c_{N} \in \mathbb{C}$ and all $x_{1}, \cdots, x_{N} \in G$, the following inequality holds:

$$
\sum_{m, n=1}^{N} c_{n} \overline{c_{m}} \phi\left(x_{m}^{-1} x_{n}\right) \geq 0
$$

This definition may be more intuitively realised as requiring the matrix

$$
\left(\phi\left(x_{m}^{-1} x_{n}\right)\right)_{i, j \leq N}=\left(\begin{array}{ccc}
\phi(e) & \cdots & \phi\left(x_{1}^{-1} x_{N}\right) \\
\vdots & \ddots & \vdots \\
\phi\left(x_{N}^{-1} x_{1}\right) & \cdots & \phi(e)
\end{array}\right)
$$

be a positive semi-definite matrix, for $x_{1}, \cdots, x_{N} \in G$. 
We shall denote by $\mathscr{P}(G)$ the set of all continuous positive-definite functions on $G$, and $\mathscr{P}_{1}(G)$ the set of all continuous positive-definite functions on $G$ satisfying $\phi(e)=1$.

Theorem 2.2.5. (Bochner's Theorem) Let $\phi$ be a continuous function on a locally compact abelian group $G$. Then $\phi$ is positive-definite if and only if there exists some nonnegative measure $M(\Gamma)$ such that

$$
\phi(x)=\int(x, \gamma) d \mu(\gamma) \quad(x \in G)
$$

The proof can be found in Rudin (pg 19). The proof is quite long, so we will exclude most of it. However, one direction is quite easy to see.

For suppose $\mu$ is a non-negative measure on $\Gamma$, and define $\phi(x)=\int(x, \gamma) d \mu(\gamma)$. Then in order to show $\phi(x)$ is positive-definite, consider $c_{1}, \cdots, c_{n} \in \mathbb{C}$, and $x_{1}, \cdots, x_{n} \in G$. We have

$$
\begin{aligned}
\sum_{i, j=1}^{n} c_{i} \overline{c_{j}} \phi\left(x_{i}-x_{j}\right) & =\sum_{i, j=1}^{n} c_{i} \overline{c_{j}} \int\left(x_{i}-x_{j}, \gamma\right) d \mu(\gamma) \\
& =\sum_{i, j=1}^{n} c_{i} \overline{c_{j}} \int\left(x_{i}, \gamma\right) \overline{\left(x_{j}, \gamma\right)} d \mu(\gamma) \\
& =\int \sum_{i, j=1}^{n} c_{i} \overline{c_{j}}\left(x_{i}, \gamma\right) \overline{\left(x_{j}, \gamma\right)} d \mu(\gamma) \\
& =\int \sum_{i, j=1}^{n} c_{i} \overline{c_{j}}\left(x_{i}, \gamma\right) \overline{\left(x_{j}, \gamma\right)} d \mu(\gamma) \\
& =\int\left(\sum_{i}^{n} c_{i}\left(x_{i}, \gamma\right)\right)\left(\sum_{j}^{n} \overline{c_{j}\left(x_{j}, \gamma\right)}\right) d \mu(\gamma) \\
& =\int\left|\sum_{i}^{n} c_{i}\left(x_{i}, \gamma\right)\right|^{2} d \mu(\gamma) \geq 0
\end{aligned}
$$

Hence the non-negative measure of $M(\Gamma)$ give rise to positive-definite functions on $G$.

Corollary 2.2.6. Let $f$ be a function on $\Gamma$. Then $f$ is a member of $B(\Gamma)$ if and only if it is the linear combination of continuous positive-definite functions on $\Gamma$

Proof. First let $f=\widehat{\mu} \in B(\Gamma)$. Then, by Jordan decomposition and the linearity of the Fourier-Stieltjes transform, we know $\widehat{\mu}=\widehat{\mu}_{1}-\widehat{\mu}_{2}+i \widehat{\mu}_{3}-i \widehat{\mu}_{4}$, which we know are continuous positive-definite functions due to Bochner's theorem.

Conversely, suppose $f=\sum_{n=1}^{N} \alpha_{n} \phi_{n}$, for $\alpha_{n} \in \mathbb{C}$ and $\phi_{n} \in \mathscr{P}(G)$. Then, again by Bochner's 
theorem, each $\phi_{n}$ is of the form $\widehat{\mu}_{n}$ for non-negative $\mu_{n}$. As such, if we define

$$
\mu:=\sum_{n=1}^{N} \alpha_{n} \mu_{n}
$$

then $\mu \in M(G)$, and $\widehat{\mu}=\sum_{n=1}^{N} \alpha_{n} \widehat{\mu}_{n}=f$, hence the result is shown.

Using this corollary, we can form a new definition for $B(\Gamma)$ that does not make reference to $\Gamma$ being the dual group of some $G$; a structure we don't have in the case that $\Gamma$ is non-abelian.

Definition 2.2.7. The Fourier-Stieltjes algebra $B(\Gamma)$ is the algebra of linear combinations of continuous positive-definite functions on $\Gamma$.

This definition is first given in Eymard's seminal paper [4]. In this paper, it is shown that one way to define the norm on $B(\Gamma)$ is using the formula

$$
\|u\|_{B(\Gamma)}=\sup \left|\sum_{n}^{N} c_{n} u\left(\gamma_{n}\right)\right|
$$

where the supremum is taken over all finite collections of $\gamma_{n} \in \Gamma, c_{n} \in \mathbb{C}$ satisfying

$$
\sup _{\phi \in \mathscr{P}_{1}\left(\Gamma_{d}\right),} \sum_{n=1}^{N} \sum_{m=1}^{N} c_{n} \overline{c_{m}} \phi\left(\gamma_{m}^{-1} \gamma_{n}\right)=1
$$

where $\Gamma_{d}$ is $\Gamma$ equipped with the discrete topology.

This new norm agrees with the standard norm on $B(\Gamma)$, in the case that $\Gamma$ is abelian.

\subsection{The Haar Measure}

In the next section, we are going to define the space of integrable functions. For this, we require a measure that connects intimately with the group and topological structure.

Definition 2.3.1. A regular $[0, \infty]$-valued measure $m$ on locally compact group $G$ is called a Haar measure if it satisfies

$$
m(x E)=m(E) \quad \text { for all } x \in G, \text { and all Borel sets } E
$$

This property is called translational invariance. In the case the $G$ is non-abelian, this is called left invariance, and the measure is called the left Haar measure. The right Haar measure is a measure with right invariance. In general, the left Haar and right Haar measures are distinct. 
Theorem 2.3.2. Every locally compact group $G$ admits both a left and right Haar measure.

The proof of this statement is quite involved, and can be found in Theorem 9.2.1 in [2]. We shall give an outline of the construction of the left Haar measure, which will be reminiscent of a technique for estimating the area of planar regions.

By taking subsets $K$ and $V$ of $G$, such that $K$ is compact, and $V$ has non-empty interior, then we can take translates $x V^{\circ}$ of $V^{\circ}$ to be an open cover of $K$. As $K$ is compact, there must exist a finite sequence $\left\{x_{1}, \cdots, x_{n}\right\}$ such that $K$ is covered by $\bigcup_{i=1}^{n} x_{i} V^{\circ}$. From here, we can define \# $(K: V)$ to be the least possible $n$ such that $n$ translates of $V^{\circ}$ cover $K$.

From here, a compact subset $K_{0}$ of $G$ with non-empty interior is chosen, to serve as a standard for measuring the size of other compact subsets. For each open neighbourhood $U$ of the identity $e \in G$, we define the function $h_{U}: \mathscr{C} \rightarrow \mathbb{R}$ by

$$
h_{U}(K)=\frac{\#(K: U)}{\#\left(K_{0}: U\right)}
$$

where $\mathscr{C}$ denotes the collection of all compact subsets of $G$. This estimates the size of $K$ in terms of $K_{0}$, by counting how many more (or fewer) translates of $U$ are needed to cover $K$. As $U$ gets smaller, the size estimate become more accurate, as there will be less overlap between translates of $U$, and less overhang (translates of $U$ covering $G \backslash K$ ).

From here, a sort of "limit" of $h_{U}$ is constructed, by considering $U$ moving along a net of open neighbourhoods of the identity, towards the empty set. This gives a function $h: \mathscr{C} \rightarrow \mathbb{R}$, which satisfies many nice conditions, such as being non-negative, having $h(\emptyset)=0, h\left(K_{0}\right)=1, h(x K)=h(K)$, and $h\left(K_{1} \cup K_{2}\right) \leq h\left(K_{1}\right)+h\left(K_{2}\right)$ (with the equality holding if $\left.K_{1} \cap K_{2}=\emptyset\right)$.

Our size estimating function $h$ is only defined on compact subsets of $G$, but can be extended to an outer measure $\mu^{*}$ on $G$. First we define $\mu^{*}$ on the open subsets of $G$ by

$$
\mu^{*}(U)=\sup \{h(K): K \in \mathscr{C}, K \subseteq U\}
$$

then further extend it to all subsets by defining

$$
\mu^{*}(A)=\inf \left\{\mu^{*}(U): U \text { is open }, A \subseteq U\right\}
$$

This indeed forms a non-zero, left translationally invariant outer measure. If we restrict this outer measure to $\mathscr{B}(G)$, the Borel sets of $G$ (the $\sigma$-algebra generated by the open sets of $G$ ), it is then a measure; the left Haar measure of $G$.

The proof holds similarly for the existence of the right Haar measure, or we can easily see that $m_{R}(E)=m_{L}\left(E^{-1}\right)$ gives a right Haar measure. In the case that $G$ is abelian, a left 
Haar measure is trivially also a right Haar measure. The following statements we shall make about the left Haar measure shall hold equally true for the right Haar measure with similar proofs.

Lemma 2.3.3. The left Haar measure of $G$ is unique up to scalar multiplication, hence referring to it as the left Haar measure is justified.

Proof. Suppose $\mu, v$ are both (nonzero) left Haar measures on $G$. Fix some nonzero $g \in C_{C}^{+}(G)$, so that $\int g m_{1}, \int g m_{2}>0$. For any $f \in C_{C}(G)$, we have

$$
\begin{aligned}
\iint \frac{f(x) g(y x)}{\int g(t x) d v(t)} d v(y) d \mu(x) & =\iint \frac{f(x) g(y x)}{\int g(t x) d v(t)} d \mu(x) d v(y) & \text { Fubini's theorem } \\
& =\iint \frac{f\left(y^{-1} x\right) g(x)}{\int g\left(t y^{-1} x\right) d v(t)} d \mu(x) d v(y) & x \rightarrow y^{-1} x \\
& =\iint \frac{f\left(y^{-1} x\right) g(x)}{\int g\left(t y^{-1} x\right) d v(t)} d v(y) d \mu(x) & \text { Fubini's theorem } \\
& =\iint \frac{f\left(y^{-1}\right) g(x)}{\int g\left(t y^{-1}\right) d v(t)} d v(y) d \mu(x) & y \rightarrow x y \\
& =\int g(x) d \mu(x) \int \frac{f\left(y^{-1}\right)}{\int g\left(t y^{-1}\right) d v(t)} d v(y) &
\end{aligned}
$$

Note that the above use of Fubini's theorem is legitimate, as $\int g(t x) d v(t)$ is continuous and vanishes nowhere, hence the fraction is also continuous. Re-examining the left hand side of the equation also shows us that

$$
\iint \frac{f(x) g(y x)}{\int g(t x) d v(t)} d v(y) d \mu(x)=\int f(x) \frac{\int g(y x) d v(y)}{\int g(t x) d v(t)} d \mu(x)=\int f(x) d \mu(x)
$$

Hence we get

$$
\int f(x) d \mu(x)=\int g(x) d \mu(x) \int \frac{f\left(y^{-1}\right)}{\int g\left(t y^{-1}\right) d v(t)} d v(y)
$$

Thus we find that the ratio between $\int f(x) d \mu(x)$ and $\int g(x) d \mu(x)$ depends purely on $f$ and $g$, not the Haar measure $\mu$ used. So we must have that

$$
\frac{\int f d \mu}{\int g d \mu}=\frac{\int f d v}{\int g d v}
$$

and so $\mu$ and $v$ differ only by a scalar. 
Hence there is (up to scalar multiplication) a unique left Haar measure, which we shall call $m$. If $G$ is discrete, it is typical to use the counting measure for the Haar measure. If $G$ is not discrete, but is compact, it is typical to normalise $m$ such that $m(G)=1$.

Example 2.3.4. The Haar measure on $\mathbb{R}$ is the length measure $m([a, b])=b-a$, although in some texts the scalar multiple $\frac{m}{\sqrt{2 \pi}}$ is used, in order to preserve Haar measure when taking the group dual. Given the above convention, the Haar measure of $\mathbb{Z}$ is the counting measure, and the Haar measure of $\mathbb{T}$ is the arc length measure, divided by a factor of $\frac{1}{2 \pi}$, to affirm that $m(\mathbb{T})=1$.

As the left Haar measures is unique, when we integrate with respect to it, we shall say $d x$ in place of $d m(x)$. If $m$ is the left Haar measure on $G$, then the measure $m_{x}(E)=$ $m(E x)$ will also be a left Haar measure and hence must differ from $m$ only by a scalar multiple. This scalar will be dependent only on $x \in G$, and hence we can define the modular function.

Definition 2.3.5. The modular function of a locally compact group $G$ is a function

$$
\Delta: G \rightarrow \mathbb{R}
$$

such that $\Delta(x) m=m_{x}$, where $m$ is the left Haar measure of $G$.

If $\mu, v$ are two left Haar measures, then $v=c \mu$ for some $c \in \mathbb{R}$. We also have $v_{x}=c \mu_{x}=$ $\Delta(x) v=c \Delta(x) \mu$, so indeed $\Delta$ is uniquely determined by the group, and not which Haar measure is being used as the standard.

By observing the equation

$$
\Delta(x y) \mu(E)=\mu(E x y)=\Delta(y) \mu(E x)=\Delta(x) \Delta(y) \mu(E)
$$

we can see that $\Delta(x y)=\Delta(x) \Delta(y)$. So in fact if $\Delta(x)>1$ for any $x \in G$, then $\Delta$ will be unbounded (consider $\Delta\left(x^{n}\right)$ as $n \rightarrow \infty$ ).

If $\Delta \equiv 1$, then $G$ is called a unimodular group. If $G$ is abelian, then it is clearly unimodular, but also if $G$ is discrete, as the left Haar measure is the counting measure, which is also right-translation invariant, hence $G$ will be unimodular.

It can be seen (such as in [5], Proposition 2.31) that $d m\left(x^{-1}\right)=\Delta\left(x^{-1}\right) d m(x)$.

\subsection{The integrable function space $L^{1}(G)$}

Definition 2.4.1. $\mathscr{L}^{p}(G)$ is the space of all functions on $G$ which are $p$-integrable with respect to the (left) Haar measure, for $1 \leq p \leq \infty$. $L^{p}(G)$ is a quotient space of $\mathscr{L}^{p}(G)$, in which $f_{1} \sim f_{2}$ if the set of points where $f_{1}$ and $f_{2}$ disagree is: 
i. null (of Haar measure zero), for $p<\infty$

ii. locally null (has null intersection with every compact set) for $p=\infty$

For $f \in L^{p}(G)$, we define $f_{x} \in L^{p}(G)$, the translate of $f$ by $x \in G$ to be $f_{x}(y)=f(y x)$. If $G$ is non-abelian, this is called the right translate, while the left translate is ${ }_{x} f(y)=f(x y)$.

Definition 2.4.2. For any Borel functions $f$ and $g$, we can define

$$
(f * g)(x)=\int f(y) g\left(y^{-1} x\right) d y
$$

to be the convolution of functions.

Convolution of functions is associative, and commutative if and only if $G$ is abelian.

The space $L^{1}(G)$ is of particular interest to us. It can be viewed as a subspace of $M(G)$, as for $f \in L^{1}(G)$, we can define a $\mu_{f} \in M(G)$ to be $\mu_{f}(E)=\int_{E} f(x) d m$. We have

$$
\left\|f_{x}\right\|_{1}=\|f\|_{1}=\left\|\mu_{f}\right\|=\left\|\mu_{f_{x}}\right\|
$$

and $\mu_{f} * \mu_{g}=\mu_{f * g}$, so $L^{1}(G)$ is in fact a Banach subalgebra of $M(G)$, when equipped with convolution of functions. In particular, $\|f * g\|_{1} \leq\|f\|_{1}\|g\|_{1}$. Moreover, $L^{1}(G)$ is a closed ideal of $M(G)$.

\subsection{The Fourier algebra $A(\Gamma)$}

Definition 2.5.1. For $f \in L^{1}(G)$, we define the Fourier Transform of $f$ to be the function

$$
\widehat{f}: \gamma \mapsto \int_{G}(-x, \gamma) f(x) d x \quad(\forall \gamma \in \Gamma)
$$

The image of $L^{1}(G)$ under the Fourier Transform is called the Fourier algebra, and is denoted $A(\Gamma)$. We equip $A(\Gamma)$ with the norm inherited from $L^{1}(G)$.

Clearly $A(\Gamma)$ is a subspace of $B(\Gamma)$, as we have $\widehat{f}=\widehat{\mu_{f}}$. In fact, $A(\Gamma) \subseteq C_{0}(\Gamma)$ also holds true, forming a more general version of the Lebesgue-Riemann lemma.

Example 2.5.2. For

1. $G=\mathbb{Z}$, we have $\widehat{f}\left(e^{i \theta}\right)=\sum_{n=-\infty}^{\infty} e^{-i n \theta} f(n)$, for $e^{i \theta} \in \mathbb{T}$

2. $G=\mathbb{T}$, we have $\widehat{f}(n)=\frac{1}{2 \pi} \int_{-\pi}^{\pi} e^{-i n \theta} f\left(e^{i \theta}\right) d \theta$, for $n \in \mathbb{Z}$

3. $G=\mathbb{R}$, we have $\widehat{f}(x)=\int_{-\infty}^{\infty} e^{-i x y} f(y) d y$, for $x \in \mathbb{R}$ 
Proposition 2.5.3. $L^{2}(G) \cdot L^{2}(G)=L^{1}(G)$, with norm defined by

$$
\|h\|_{1}=\inf \left\{\|f\|_{2}\|g\|_{2}: h=f g, f, g \in L^{2}(G)\right\}
$$

Proof. Let $f, g$ be in $L^{2}(G)$. Then $\int|f|^{2} d m$ and $\int|g|^{2} d m$ are both finite values. Thus

$$
\int_{G}|f g| d m \leq\left(\int_{G}|f|^{2} d m\right)^{\frac{1}{2}}\left(\int|g|^{2} d m\right)^{\frac{1}{2}}
$$

by the Cauchy-Schwarz inequality, so indeed $L^{2}(G) \cdot L^{2}(G) \subseteq L^{1}(G)$. Conversely, any $h \in L^{1}(G)$ can be expressed as $h^{\frac{1}{2}} \cdot h^{\frac{1}{2}} \in L^{2}(G) \cdot L^{2}(G)$, so we are done.

An interesting consequence of this is that $L^{2}(\Gamma) * L^{2}(\Gamma)=A(\Gamma)$. In order to show this, we will need to harness what is called the Plancherel Theorem. This states that the Fourier transform, when restricted to the space of functions in both $L^{1}(G)$ and $L^{2}(G)$, is an isometry (with respect to the $L^{2}$ norm) onto a dense subspace of $L^{2}(\Gamma)$. As such, it can be extended uniquely to an isometry from $L^{2}(G)$ onto $L^{2}(\Gamma)$. This isometry is called the Plancherel transform, and from it we can obtain the Parseval formula, which states

$$
\int_{G} f(x) \overline{g(x)} d x=\int_{\Gamma} \widehat{f}(\gamma) \bar{g}(\gamma) d \gamma \quad f, g \in L^{2}(G)
$$

The proof of these statements can be found in [9].

Proposition 2.5.4. The Fourier transform maps $L^{2}(G) \cdot L^{2}(G)$ onto $L^{2}(\Gamma) * L^{2}(\Gamma)$, hence $L^{2}(\Gamma) * L^{2}(\Gamma)=A(\Gamma)$

Sketch of proof. Let $f, g \in L^{1}(G) \cap L^{2}(G)$. If we use the Parseval formula with $\bar{g}$ in place of $g$, then as the Fourier transform of $\bar{g}$ is $\int(-x, \gamma) \overline{g(x)} d x$ (which has complex conjugate $\left.\int(-x,-\gamma) g(x) d x=\widehat{g}(-\gamma)\right)$, we get

$$
\int f(x) g(x) d x=\int \widehat{f}(\gamma) \widehat{g}(-\gamma) d \gamma
$$

Further, if for some $\gamma_{0} \in \Gamma$, we use the Parseval formula with $\left(-x, \gamma_{0}\right) \overline{g(x)}$ instead of $g(x)$, we get

$$
\int f(x) g(x)\left(-x, \gamma_{0}\right) d x=\int \widehat{f}(\gamma) \widehat{g}\left(\gamma_{0}-\gamma\right) d \gamma
$$

which is the value of $\widehat{f} * \widehat{g}$ evaluated at $\gamma_{0}$.

These results can be extended to $f, g \in L^{2}(G)$ (and not necessarily in $L^{1}(G)$ ), so indeed for any $h \in L^{1}(G)$, we can express $h$ as $f g$ for $f, g \in L^{2}(G)$, which will have Fourier transform $\widehat{h} \in A(\Gamma)$ satisfying $\widehat{h}=\widehat{f} * \widehat{g} \in L^{2}(\Gamma)$.

In the case that $\Gamma$ is a non-abelian group, it seems desirable to define $A(\Gamma)$ to simply be $L^{2}(\Gamma) * L^{2}(\Gamma)$, with the norm defined by

$$
\|\phi\|_{A(\Gamma)}:=\inf \left\{\|f\|_{2}\|g\|_{2}: \phi=f * g, f, g \in L^{2}(\Gamma)\right\}
$$


However, the convolution $f * g$ for $f, g \in L^{2}(\Gamma)$ is only guaranteed to be well-defined when $\Gamma$ is unimodular, such as when $\Gamma$ is abelian, or discrete.

Consider briefly the case when $\Gamma$ is non-unimodular, and take $f, g \in L^{2}(\Gamma)$. Then $f *$ $g(\gamma)=\int f(\eta) g\left(\eta^{-1} \gamma\right) d \eta$. Consider $f * g(e)=\int f(\eta) g\left(\eta^{-1}\right) d \eta$. By Hölder's inequality, we have

$$
|f * g(e)|=\left|\int f(\eta) g\left(\eta^{-1}\right) d \eta\right| \leq\left(\int|f(\eta)|^{2} d \eta\right)^{\frac{1}{2}}\left(\int|\check{g}(\eta)|^{2} d \eta\right)^{\frac{1}{2}}
$$

where $\check{g}(\eta)=g\left(\eta^{-1}\right)$.

For $f * g(1)$ to be well defined, ideally we would have $\check{g} \in L^{2}(\Gamma)$, however

$$
\int|\check{g}(\eta)|^{2} d \eta=\int \Delta(\eta)^{-1}|g(\eta)|^{2} d \eta
$$

As $\Gamma$ is unimodular, $\Delta(\eta)^{-1}$ is unbounded, so it is not necessary that $\check{g} \in L^{2}(\Gamma)$ when $g \in L^{2}(\Gamma)$, and so the construction above may not work in general.

As such, we need a different way to define $A(\Gamma)$ in the non-abelian case, which will work even if $\Gamma$ is not unimodular.

This problem was investigated by Eymard in [4], and it is shown that we can define $A(\Gamma)$ as follows.

Definition 2.5.5. The Fourier algebra $A(\Gamma)$ of a locally compact group $\Gamma$ is the set

$$
A(\Gamma)=L^{2}(\Gamma) * \overline{L^{2}(\Gamma)}
$$

with the norm $\|\phi\|=\inf \left\{\|f\|_{2}\|g\|_{2}: \phi=f * \check{g}, f, g \in L^{2}(\Gamma)\right\}$.

This may seem unbelievable that this is indeed an algebra (or even a linear space), but it follows more easily after we observe other ways to construct $A(\Gamma)$. If we let $\widetilde{g}(\gamma):=$ $\bar{g}(\gamma)=\overline{g\left(\gamma^{-1}\right)},[4]$ shows the following 1 .

Theorem 2.5.6. The complex vector spaces of functions

i. $E_{1} \quad$ generated by $f * \check{g}$ for $f, g \in C_{C}(\Gamma)$

ii. $E_{2} \quad$ generated by $h * \widetilde{h}$ for $h \in C_{C}(\Gamma)$

iii. $E_{3} \quad$ generated by $f * \check{g}$ for $f, g \in L^{\infty}(\Gamma)$ with compact support

iv. $E_{4} \quad$ generated by $h * \widetilde{h}$ for $h \in L^{\infty}(\Gamma)$ with compact support

\footnotetext{
${ }^{1}$ Actually, we are presenting Eymard's results in a different order to fit our purpose. In [4], the statement of Theorem 2.5.6 is used as justification to define $A(\Gamma)=L^{2}(\Gamma) * \widehat{L^{2}(\Gamma)}$, and so the definition given in 2.5.5 is given after this theorem.
} 

v. $E_{5}=B(\Gamma) \cap C_{C}(\Gamma)$
vi. $E_{6} \quad$ generated by $\mathscr{P}(\Gamma) \cap C_{C}(\Gamma)$
vii. $E_{7} \quad$ generated by $u \in \mathscr{P}(\Gamma)$ satisfying $\Delta^{-\frac{1}{2}} u \in L^{1}(\Gamma)$
viii. $E_{8} \quad$ generated by $\mathscr{P}(\Gamma) \cap L^{2}(\Gamma)$
ix. $E_{9} \quad$ generated by $h * \widetilde{h}$ for $h \in L^{2}(\Gamma)$
x. $E_{10} \quad$ generated by $f * \check{g}$ for $f, g \in L^{2}(\Gamma)$

all have $A(\Gamma)$ as their closure in $B(\Gamma)$, and the $A(\Gamma)$ norm agrees with the inherited norms.

Clearly, the space $A(\Gamma)$ is an algebra, and is closed under translation.

Theorem 2.5.7. $A(\Gamma)$ is a dense subalgebra of $C_{0}(\Gamma)$.

Proof. It is now obvious that $A(\Gamma)$ is a subalgebra of $C_{0}(\Gamma)$, so we aim to use the StoneWeierstrass theorem to prove it is dense.

For any $\gamma_{0} \in \Gamma$, we can define a function $f \in L^{2}(\Gamma)$ that is 1 on a non-empty compact neighbourhood $C$ of $\gamma_{0}$, and 0 otherwise. Recall that $\gamma_{0} f(\gamma)=f\left(\gamma_{0} \gamma\right)$, and so $\widetilde{\gamma_{0} f}(\gamma)=$ $\overline{f\left(\gamma_{0} \gamma^{-1}\right)}$. Then

$$
\left.f * \widetilde{\gamma_{0} f}\left(\gamma_{0}\right)=\int f(\gamma) \widetilde{\left(\widetilde{\gamma_{0}} f\right.}\left(\gamma^{-1} \gamma_{0}\right)\right) d \gamma=\int f(\gamma) \overline{\gamma_{0} f\left(\gamma_{0}^{-1} \gamma\right)} d \gamma=\int|f(\gamma)|^{2} d \gamma=m(C)
$$

where $m(C)$ is the (non-zero) Haar measure of $C$. Hence $f * \widetilde{\gamma_{0} f}$ (which can also be viewed as $\left.f * \overline{\gamma_{0} f}\right)$ is in $A(\Gamma)$ and maps $\gamma_{0}$ to some nonzero value, therefore $A(\Gamma)$ vanishes nowhere.

Now let $\gamma_{0} \neq \gamma_{1} \in \Gamma$. As $A(\Gamma)$ is translation invariant, we can assume $\gamma_{1}$ is the identity, and $\gamma_{0}$ is any other element. The goal will be to construct a function $f$, such that $f * f\left(\gamma_{0}\right)=0$, and $f * f(e) \neq 0$. To do this, we will construct our $f$ to have support $U$, such that $\gamma_{0}$ is not contained in $U^{2}$.

Let $A$ be an open neighbourhood of $e$ whose closure does not contain $\gamma_{0}$ (this is possible, as we require locally compact groups to be Hausdorff). By the continuity of the inverse operation $t \mapsto t^{-1}$, we know that $A^{-1}$ is open also, and so $B=A \cap A^{-1}$ is an open neighbourhood of $e . B$ is called a symmetric neighbourhood of $e$, as it is closed under inverses. The pre-image of $B$ under the group product is a neighbourhood of $\langle e, e\rangle \in \Gamma \times \Gamma$, and hence contains some $U \times U \subseteq \Gamma \times \Gamma$, where $U$ contains $e$, does not contain $\gamma_{1}$ in its closure, and has $U^{2} \subseteq B$. Hence if we choose $f$ to be the characteristic function of $U, f * \widetilde{f}$ will evaluate $e$ to be $m(U) \neq 0$, and $\gamma_{0}$ to be 02 .

\footnotetext{
${ }^{2}$ We have assumed that $U$ has non-zero Haar measure. This is true for all non-empty open sets, as the Haar measure is regular, hence we can find some compact set $C$ such that $m(C) \neq 0$, and $n$ many translates of $U$ cover $C$. Therefore $m(U) \geq \frac{1}{n} m(C)$
} 
Finally, if $f * \check{g} \in A(\Gamma)$, then $\overline{f * \check{g}} \in A(\Gamma)$, as $\bar{f} * \bar{g}=\overline{f * g}$. 


\section{Chapter 3}

\section{Idempotents of small norm on LCA groups}

Throughout this chapter, we will use $G$ to denote a locally compact abelian group with dual group $\Gamma$. We shall use + to denote the abelian group operation, and 0 to denote the identity element of both groups. Subscripts will be used in case of ambiguity.

\subsection{Idempotent measures}

Definition 3.1.1. A member $a$ of an algebra $A$ is called an idempotent if $a^{2}=a$. In particular, we say a measure $\mu \in M(G)$ is idempotent if $\mu * \mu=\mu$. We denote the set of all idempotent measures on $G$ by $J(G)$.

If $\mu \in M(G)$ is an idempotent measure, then the Fourier-Stieltjes transform $\widehat{\mu}$ must be an idempotent function in $B(\Gamma) ; \widehat{\mu}$ only takes values 0 and 1 . This means that $\widehat{\mu}$ is actually a characteristic function for some subset of $\Gamma$. We shall call this subset $S(\mu)$, and more formally define it to be

$$
S(\mu)=\{\gamma \in \Gamma: \widehat{\mu}(\gamma)=1\}
$$

When it comes to discussing norms of idempotent measures, there are some small but important details we can use. Consider $\mu \in J(G)$. As $\mu$ is idempotent, $\|\mu\|=\|\mu * \mu\| \leq$ $\|\mu\|^{2}$, so if $\mu \neq 0$, then $\|\mu\| \geq 1$. So the smallest norm of interest to us will be $\|\mu\|=1$. A very important fact we will prove shortly is that idempotent measures have norm 1 if and only if $S(\mu)$ is an open coset in $\Gamma$, but first we need this small result.

Proposition 3.1.2. Let $\phi$ be a positive definite function on $\Gamma$. Then for all $\gamma, \eta \in \Gamma$,

$$
|\phi(\gamma)-\phi(\eta)|^{2} \leq 2 \phi(0) \operatorname{Re}[\phi(0)-\phi(\gamma-\eta)]
$$

Proof. Let $c_{1}:=1$, and set 


$$
c_{2}:=\frac{c|\phi(\gamma)-\phi(\eta)|}{\phi(\gamma)-\phi(\eta)}, \quad c_{3}:=-c_{2}
$$

for real valued $c$. Then, by the definition of $\phi$ being positive definite, with $0, \gamma, \eta$ as our $x_{1}, x_{2}, x_{3}$ and $c_{1}, c_{2}, c_{3} \in \mathbb{C}$ as our constants, we must have

$$
\phi(0)\left(1+2 c^{2}\right)+2 c|\phi(\gamma)-\phi(\eta)|-2 c^{2} \operatorname{Re} \phi(\gamma-\eta) \geq 0
$$

which viewed as a quadratic polynomial in $c$ can't possibly have a non-negative discriminant, thus giving us the result.

Theorem 3.1.3. For non-zero $\mu \in J(G),\|\mu\|=1$ if and only if $S(\mu)$ is an open coset in $\Gamma$.

Proof. If we suppose that $\|\mu\|=1$, then $\mu$ is non-zero, and hence $S(\mu)$ is non-empty. By taking $\gamma_{0} \in S(\mu)$, we can define a measure $d v(x)=\left(x,-\gamma_{0}\right) d \mu(x)$, to ensure that $0 \in S(v)$. With this, we have

$$
1=\widehat{v}\left(0_{\Gamma}\right)=\int d v=v(G) \leq\|v\|=\|\mu\|=1
$$

Hence $v(G)=\|v\|=1$. This can only happen if $v$ is a non-negative measure, and hence by Bochner's Theorem, if $\widehat{v}$ is a positive definite function. With this, we are now ready to show that $S(v)$ is an subgroup, and hence $S(\mu)$ is the coset $\gamma+S(v)$ in $\Gamma$.

We have defined $v$ such that $S(v)$ contains the identity. Let $\gamma \in S(v)$ be arbitrary. Then

$$
\int(x,-\gamma) d v(x)=\int \overline{(x, \gamma)} d v(x)=\overline{\int(x, \gamma) d v(x)}=1
$$

so $-\gamma \in S(v)$ also.

As we have shown $\widehat{v}$ is positive-definite, the inequality in 3.1 .2 holds. Hence for $\gamma_{1}, \gamma_{2} \in$ $S(v)$, since we have $-\gamma_{1},-\gamma_{2} \in S(v)$, and by setting $\gamma:=\gamma_{1}-\gamma_{2}, \eta:=\gamma_{1}$, we get

$$
\left|\widehat{v}\left(\gamma_{1}-\gamma_{2}\right)-\widehat{v}\left(\gamma_{1}\right)\right| \leq 2 \widehat{v}\left(0_{\Gamma}\right) \operatorname{Re}\left(\widehat{v}\left(0_{\Gamma}\right)-\widehat{v}\left(-\gamma_{2}\right)\right)=0
$$

So we must have $\widehat{v}\left(\gamma_{1}-\gamma_{2}\right)=\widehat{v}\left(\gamma_{1}\right)=1$, hence $\gamma_{1}-\gamma_{2} \in S(v)$, completing the proof that $S(v)$ is an subgroup in $\Gamma$.

To prove the converse, assume $S(\mu)$ is a open coset in $\Gamma$. Then there is some open subgroup $\Lambda$ and some $\gamma_{0} \in \Gamma$ such that $\gamma_{0}+\Lambda=S(\mu)$. Let $H$ be the annihilator of $\Lambda$. Note that by Corollary 1.3.4, $H$ is be compact. Construct a measure $v$ by setting $d v(x)=\left(x,-\gamma_{0}\right) d m_{H}(x)$, for $m_{H}$ the Haar measure of $H$, normalised to have $m_{H}(H)=1$. 1

\footnotetext{
${ }^{1}$ This may appear to be nonsensical, as $m_{H}$ is not a member of $M(G)$, but any measure $\mu \in M(H)$ can be extended to one in $M(G)$ by considering it as some $\widetilde{\mu} \in M(G)$ such that $\widetilde{\mu}(E)=\mu(E \cap H)$
} 
For any $\gamma \in \Gamma, \widehat{v}(\gamma)=\int\left(x, \gamma-\gamma_{0}\right) d m_{H}(x)$. First notice that if $\gamma$ is such that $\gamma-\gamma_{0} \in \Lambda$, then $\widehat{v}(\gamma)=\int\left(x, \gamma-\gamma_{0}\right) d m_{H}(x)=\int d m_{H}=1$. Now consider when $\gamma-\gamma_{0}$ is not in $S(\mu)$. $\left(x, \gamma-\gamma_{0}\right)$ will not be 1 for all $x \in H$, otherwise $\gamma-\gamma_{0}$ would be in the $\Lambda$, so there must exist some $x_{0} \in H$ such that $\left(x_{0}, \gamma-\gamma_{0}\right) \neq 1$. This means

$\widehat{v}(\gamma)=\int\left(x, \gamma-\gamma_{0}\right) d m_{H}(x)=\int\left(x_{0}+x, \gamma-\gamma_{0}\right) d m_{H}(x)=\left(x_{0}, \gamma-\gamma_{0}\right) \int\left(x, \gamma-\gamma_{0}\right) d m_{H}(x)$

thus $\int\left(x, \gamma-\gamma_{0}\right) d m_{H}(x)=\left(x_{0}, \gamma-\gamma_{0}\right) \int\left(x, \gamma-\gamma_{0}\right) d m_{H}(x)$, which can only happen if they are both zero.

Hence $\widehat{\mu}=\widehat{v}$, as their supports are identical. Ergo, by Fourier Uniqueness, we have $\mu=v$. By construction, $d v(x)=\left(x,-\gamma_{0}\right) d m_{H}(x)$, so

$$
\|\mu\|=\|v\|=|v|(H)=\int\left|\left(x,-\gamma_{0}\right)\right| d m(H)=1
$$

Proposition 3.1.4. Let $\mu \in M(G)$ be of the form $d \mu(x)=\left[\left(-x, \gamma_{1}\right)+\left(-x, \gamma_{2}\right)\right] d m(x)$, for distinct $\gamma_{1}, \gamma_{2} \in \Gamma$ and $m$ the Haar measure of $G$. Then $\mu$ is idempotent, and will have norm defined by

$$
\|\mu\|= \begin{cases}2 /\left(q \sin \left(\frac{\pi}{2 q}\right)\right) & (\text { for odd } q) \\ 2 /\left(q \tan \left(\frac{\pi}{2 q}\right)\right) & (\text { for even } q) \\ \frac{4}{\pi} & (q=\infty)\end{cases}
$$

where $q \geq 3$ is the order of $\gamma_{2}^{-1} \gamma_{1}$ in $\Gamma$.

This is not hard to calculate, but the details can be found in [11]

\subsection{Idempotents of norm less than $\frac{4}{3}$}

Let us now assume that $\mu$ is an idempotent measure of $M(G)$, for compact abelian $G$, such that $\|\mu\|<\frac{4}{3}$. We will write $S$ for $S(\mu)$.

Lemma 3.2.1. Suppose there exist $u, v \in S$ and $w \in \Gamma$ such that $u+w \in S$, but both $v+w, v-w \notin S$. Then we have $\|\mu\| \geq \frac{4}{3}$

Proof. In order to prove this, we harness the fact that for any $f \in L^{\infty}(G)$, we have

$$
\left|\int_{G} f d \mu\right| \leq\|f\|_{\infty}\|\mu\|
$$


Now it suffices to find such an $f$ to satisfy

$$
\frac{4}{3} \leq \frac{\left|\int_{G} f d \mu\right|}{\|f\|_{\infty}} \leq\|\mu\|
$$

We define $f$ to be the function

$$
\begin{aligned}
f(x) & =2(-x, u)+2(-x, v)+2(-x, u+w)+\frac{1}{2}(-x, u-w)-(-x, v+w)-(-x, v-w) \\
& =(-x, u)\left[2+2(-x, w)+\frac{1}{2}(-x,-w)\right]+(-x, v)[2-(-x, w)-(-x,-w)]
\end{aligned}
$$

From the first form of $f$ given above, we have

$$
\left|\int_{G} f(x) d \mu(x)\right|= \begin{cases}\left|2+2+2+\frac{1}{2}\right| & =\frac{13}{2} \text { If } u-w \in S(\mu) \\ |2+2+2| & =6 \text { If } u-w \notin S(\mu)\end{cases}
$$

telling us $\left|\int_{G} f d \mu\right|$ will always be at least 6 . Now, by rewriting $(-x, w)$ as $e^{i \theta}$, the second form gives us

$$
\begin{aligned}
\|f\|_{\infty} & \leq\left|2+2 e^{i \theta}+\frac{1}{2} e^{-i \theta}\right|+\left|2-e^{i \theta}-e^{-i \theta}\right| \\
& =\left|2+\frac{5}{2} \cos (\theta)+\frac{3}{2} i \sin (\theta)\right|+|2-2 \cos (\theta)| \quad(\forall \theta \in[0,2 \pi])
\end{aligned}
$$

If we examine this further, using the identity $|z|=\sqrt{z \bar{z}}$, we find

$$
\begin{aligned}
\left|2+\frac{5}{2} \cos (\theta)+\frac{3}{2} i \sin (\theta)\right|+|2-2 \cos (\theta)| & =\sqrt{\frac{25}{4}+10 \cos (\theta)+4 \cos ^{2}(\theta)}+2-2 \cos (\theta) \\
& =2 \sqrt{\left(\cos (\theta)+\frac{5}{4}\right)^{2}}+2-2 \cos (\theta) \\
& =2 \cos (\theta)+\frac{5}{2}+2-2 \cos (\theta) \\
& =\frac{9}{2}
\end{aligned}
$$

So we have $\|f\|_{\infty} \leq \frac{9}{2}$.

Hence $\frac{12}{9}=\frac{4}{3} \leq \frac{\left|\int_{G} f(x) d \mu(x)\right|}{\|f\|_{\infty}} \leq\|\mu\|$. 
Lemma 3.2.2. Let $\mu$ be an idempotent measure with $\|\mu\|<\frac{4}{3}$, and let $d$ be the difference of two elements in $S$. Then for any $c \in S$, at least one of $c \pm d$ is in $S$.

Proof. Using the above Lemma, we see that if there exists some $u, v \in S(\mu), w \in \Gamma$ such that $u+w \in S, v \pm w \notin S$, then $\|\mu\| \geq \frac{4}{3}$.

Suppose $c \in S$, and $d=b-a$ for $a, b \in S$. If $c \pm(b-a)$ are both not in $S$, then we can use $u=a, v=c, w=b-a$ to see that $\|\mu\| \geq \frac{4}{3}$. This contradicts our assumption that $\|\mu\|<\frac{4}{3}$, so indeed at least one of $c \pm(b-a) \in S$.

Corollary 3.2.3. For all possible choices of $a, b, c \in S$, we must have at least 2 of $-a+$ $b+c, a-b+c, a+b-c$ in $S$.

Proof. For any possible pairing of these sums, we have at least one must be in $S$ by Lemma 3.2.2. We can repeat Lemma 3.2.2 with the remaining two, and see at least a second must also be in $S$.

Proposition 3.2.4. If there exists some progression $\eta, \eta+\gamma, \eta+2 \gamma \in S$, then the coset $\eta+\langle\gamma\rangle$ must be completely contained inside $S$.

Proof. Assume $\eta, \eta+\gamma, \eta+2 \gamma \in S$. Using Lemma 3.2.2 with $c=\eta+\gamma, d=2 \gamma$, we see we must have one of $\eta+3 \gamma, \eta-\gamma \in S$. Assume WLOG that we have $\eta+3 \gamma \in S$. We now aim to show that we must have that also $\eta-\gamma \in S$. Let us assume not, and derive a contradiction.

Consider the function $f(x)=-\frac{3}{2}(-x, \eta-\gamma)+3(-x, \eta)+(-x, \eta+\gamma)+(-x, \eta+2 \gamma)+$ $(-x, \eta+3 \gamma)$.

We have $\left|\int_{G} f d \mu\right|=6$, and can evaluate $\|f\|_{\infty}$ using the relation $|z|=\sqrt{z \bar{z}}$. Let us write $(-x, \gamma)$ as $e^{i t}$, and remove the factor of $(-x, \eta)$. This gives us

$$
|f(x)|=\left|-\frac{3}{2} e^{-i t}+3+e^{i t}+e^{2 i t}+e^{3 i t}\right|=\cos (t)+5 \cos (2 t)+3 \cos (3 t)-3 \cos (4 t)+\frac{57}{4}
$$

By substituting $y=\cos (t)$, we get the function $g(y)=-24 y^{4}+12 y^{3}+34 y^{2}-8 y+\frac{25}{4}$, which attains its extrema when $g^{\prime}(y)=-4(y-1)\left(24 y^{2}+15 y-2\right)=0$.

Clearly there is an extreme value at $y=1$, which is $g(1)=\frac{81}{4}$. If we now translate the function $g(y)$ downwards by $\frac{81}{4}$, we get $g(y)-\frac{81}{4}=-2(y-1)^{2}\left(12 y^{2}+18 y+7\right)$. This function is positive nowhere, so must have its global maximum at $g(y)-\frac{81}{4}=0$, when $y=1$. Hence the global maximum of $g(y)$ is precisely $\frac{81}{4}$, and by taking the square root of this value, we find $\|f\|_{\infty}=\frac{9}{2}$, so $\|\mu\| \geq \frac{12}{9}=\frac{4}{3}$, a contradiction. 
Lemma 3.2.5. If for some $\eta_{1}, \gamma \in S$ the coset $\eta_{1}+\langle\gamma\rangle$ is contained in $S$, then for any $\eta_{2} \in S$, the coset $\eta_{2}+\langle\gamma\rangle$ is also contained in $S$.

Proof. First let us translate $S$ such as to assume $\eta_{2}=0 \in S$. This has no effect on the norm of $\|\mu\|$.

Applying Lemma 3.2.2 with $c=0, d=\gamma$ and with $c=0, d=2 \gamma$ tells us we must have at least one of $\pm \gamma \in S$, and at least one of $\pm 2 \gamma \in S$. If $\gamma, 2 \gamma \in S$ or $-\gamma,-2 \gamma \in S$, then we can apply Proposition 3.2.4, and we are done. Otherwise, we can say, WLOG, that $-\gamma, 2 \gamma \in S$. Let us assume also that $\gamma,-2 \gamma \notin S$, and aim for a contradiction.

Applying Corollary 3.2 .3 with $-\gamma, 0,2 \gamma$ tells us at least two of $3 \gamma, \gamma,-3 \gamma$ are in $S$. As we are assuming $\gamma \notin S$, we are forced to conclude that $3 \gamma,-3 \gamma \in S$, and in fact, we have the progression $-3 \gamma, 0,3 \gamma \in S$, and so by 3.2.4, $\langle 3 \gamma\rangle \subseteq S$.

If we restrict $\mu$ to $\langle\gamma\rangle$, we can bind its norm $\|\mu\|$ below by considering the natural homomorphism $\phi:\langle\gamma\rangle \rightarrow\langle\gamma\rangle /\langle 3 \gamma\rangle=\mathbb{Z}_{3}$. $\|\mu\|$ only decreases when considering it as a measure on a subgroup of $G$, and as the map $f \mapsto \int_{G} f(\phi(x)) d \mu(x)$ is a bounded linear functional on $C\left(\mathbb{Z}_{3}\right)$, by Riesz Representation Theorem, there exists a unique measure $v$ on $\mathbb{Z}_{3}$ with $\|v\| \leq\|\mu\|$, such that $\int_{G} f(\phi(x)) d \mu(x)=\int_{\mathbb{Z}_{3}} f d v$.

$v$ is still idempotent, and $S(v)$ consists of precisely two elements, 0 and an element of order 3. Via 3.1.4, we can see that $v$ must have norm $\frac{2}{3 \sin (\pi / 6)}=\frac{4}{3}$, so indeed our $\mu$ must have norm $\|\mu\| \geq\|v\|=\frac{4}{3}$, the desired contradiction.

Hence we must have $\gamma$ or $-2 \gamma \in S$, and thus the whole subgroup $\langle\gamma\rangle \subseteq S$ (or upon inversing the translation, the coset $\eta_{2}+\langle\gamma\rangle \subseteq S$ ).

Corollary 3.2.6. For $\eta_{1}+\left\langle\gamma_{1}\right\rangle \subseteq S$ and $\eta_{2}+\left\langle\gamma_{2}\right\rangle \subseteq S$, the set $\eta_{1}+\left\langle\gamma_{1}\right\rangle+\left\langle\gamma_{2}\right\rangle$ is contained in $S$.

Proof. By Lemma 3.2.5, as $\eta_{1}+n \gamma_{1} \in S$ for all $n \in \mathbb{Z}$, each coset $\eta_{1}+n \gamma_{1}+\left\langle\gamma_{2}\right\rangle$ is contained in $S$.

Corollary 3.2.7. The set $\Lambda=\{\gamma \in S: \exists \eta \in S$ such that $\eta+\langle\gamma\rangle \subseteq S\}$ is a subgroup in $\Gamma$.

Proof. For $\gamma_{1}, \gamma_{2} \in \Lambda$, there exists $\eta_{1}, \eta_{2} \in S$ such that $\eta_{1}+\left\langle\gamma_{1}\right\rangle \subseteq S$ and $\eta_{2}+\left\langle\gamma_{1}\right\rangle \subseteq S$. Hence $\eta_{1}+\left\langle\gamma_{1}\right\rangle+\left\langle\gamma_{2}\right\rangle \subseteq S$, and $\eta_{1}+\left\langle\gamma_{1}+\gamma_{2}\right\rangle \subseteq \eta_{1}+\left\langle\gamma_{1}\right\rangle+\left\langle\gamma_{2}\right\rangle$, so we can conclude that $\gamma_{1}+\gamma_{2} \in \Lambda$.

As $\Lambda$ clearly contains inverses, it is a subgroup of $\Gamma$. 
Theorem 3.2.8. Let $G$ be a locally compact abelian group, and $\mu \in J(G)$. If $1<\|\mu\|<\frac{4}{3}$, then $S(\mu)$ can be written as the union of two distinct cosets of a subgroup $\Lambda$ of $\Gamma$. In particular

$$
d \mu(x)=\left[\left(-x, \gamma_{0}\right)+\left(-x, \gamma_{1}\right)\right] d m_{H}(x)
$$

where $H$ is a compact subgroup of $G$, and $\gamma_{0} \neq \gamma_{1}$ are characters of $H$.

Proof. We know $\Lambda$, as constructed above, is a subgroup of $\Gamma$. By use of Lemma 3.2.5 and Corollary 3.2.7, we see that $S$ must be the union of some collection of cosets of $\Lambda$. Assume there are more than 2 distinct cosets $\left(\gamma_{j}+\Lambda\right)$ such that $S=\bigcup_{j=1}^{n}\left(\gamma_{j}+\Lambda\right)$. Then, in particular, we can get $\gamma_{1}, \gamma_{2}, \gamma_{3} \in S$ such that $\gamma_{1}+\Lambda, \gamma_{2}+\Lambda \gamma_{3}+\Lambda$ are all disjoint.

Using Corollary 3.2.3 with $a=\gamma_{1}, b=\gamma_{2}, c=\gamma_{3}$, we can assume without loss of generality that $\gamma_{1}-\gamma_{2}+\gamma_{3}, \gamma_{1}+\gamma_{2}-\gamma_{3} \in S$. This, together with $\gamma_{1}$, forms a progression of 3 , so we must have $\gamma_{1}+\left\langle\gamma_{2}-\gamma_{3}\right\rangle \subseteq S$, hence $\gamma_{2}-\gamma_{3}, \gamma_{3}-\gamma_{2}$ must be members of $\Lambda$ itself. This means that $\gamma_{2}+\Lambda=\gamma_{3}+\Lambda$, which contradicts our assumption, so we conclude there can be no more than two distinct cosets of $\Lambda$ forming $S$.

If $S$ consisted of just 1 coset, then $\|\mu\|=1$, so it must consist of precisely 2 cosets. Hence $d \mu(x)=\left[\left(-x, \gamma_{1}\right)+\left(-x, \gamma_{2}\right)\right] d m_{H}(x)$, where $H$ is the annihilator of $\Lambda$.

Theorem 3.2.9. Let $\Gamma$ be a locally compact abelian group, and $f \in B(\Gamma)$ be an idempotent function. If $1<\|f\|<\frac{4}{3}$, then the support of $f$ is the union of two distinct cosets of a subgroup $\Lambda$ of $\Gamma$.

The interval $\left(1, \frac{4}{3}\right)$ is sharp. This follows from Proposition 3.1.4, and cannot be extended to $\left(1, \frac{4}{3}\right]$, as these theorems would not hold true with $\|\mu\|=\frac{4}{3}$. We can see this using an argument from [11], which considers $\mu$ with $S(\mu)=\{0, \gamma, 2 \gamma\}$, for $\gamma$ being an element of $\Gamma$ with order 6 . This gives $\|\mu\|=\frac{4}{3}$, while having $S(\mu)$ be the union of 3 cosets. 


\section{Chapter 4}

\section{Completely bounded Schur multipliers}

\subsection{Multipliers, and completely bounded multipliers}

When we are dealing with an amenable group $\Gamma$, the space $B(\Gamma)$ can be seen as a Pontryagin dual object of $M(\Gamma)$. However, when $\Gamma$ is non-amenable, there are various objects that can be viewed as a Pontryagin dual object of $M(\Gamma)$. One such object is the collection of completely bounded Schur multipliers on $A(\Gamma)$, denoted $M_{c b} A(\Gamma)$

Definition 4.1.1. A function $\varphi: \Gamma \rightarrow \mathbb{C}$ is a multiplier of $A(\Gamma)$ if $\varphi \cdot \psi \in A(\Gamma)$, for all $\psi \in A(\Gamma)$. The collection of all multipliers of $A(\Gamma)$ is denoted $M A(\Gamma)$.

Note that every $\varphi \in M A(\Gamma)$ must be continuous, as each $\psi \in A(\Gamma)$ is continuous, and $\varphi \cdot \psi \in A(\Gamma)$ is continuous. So

$$
\varphi=\frac{\varphi \cdot \psi}{\psi}
$$

is easily continuous at all $\gamma_{0} \in \Gamma$ when $\psi\left(\gamma_{0}\right) \neq 0$. As there must exist some $\psi$ that is nonzero for any given $\gamma_{0}, \varphi$ must be continuous everywhere on $\Gamma$.

If $\varphi$ is a multiplier of $A(\Gamma)$, then we can use it to create the multiplication map $m_{\varphi}$ : $A(\Gamma) \rightarrow A(\Gamma)$, defined by $\psi \mapsto \varphi \cdot \psi$. Using this, we can define the multiplier norm to be $\|\varphi\|_{M}:=\left\|m_{\varphi}\right\|$, where $\left\|m_{\varphi}\right\|$ is the standard operator norm.

We know $A(\Gamma)$ is an ideal in $B(\Gamma)$ (this follows from (v) in Theorem 2.5.6 that $A(\Gamma)=$ $B(\Gamma) \cap C_{C}(\Gamma)$ ), and as such, we must have that $B(\Gamma) \subseteq M A(\Gamma)$. Moreover, the inclusion from $B(\Gamma)$ into $M A(\Gamma)$ is easily seen to be norm decreasing, giving

$$
\|\cdot\|_{M} \leq\|\cdot\|_{B(\Gamma)}
$$

This means that idempotents of $B(\Gamma)$ with small norm do indeed correspond with idempotents of small(er) norm in $M A(\Gamma)$, yet this decrease in norm is too significant for the problems we aim to solve. Instead, we wish to use what is called the completely bounded 
multiplier norm. This norm is constructed by building an operator space structure on $A(\Gamma)$.

Given a Banach space $V$, an operator space structure on $V$ is a sequence of norms $\|\cdot\|_{n}$, for $n \in \mathbb{N}$, defined respectively on the $n \times n$ matrix spaces $\mathbb{M}_{n}(V)$. The norm $\|\cdot\|_{1}$ must agree with $\|\cdot\|_{V}$, and the norms $\|\cdot\|_{n}$ must satisfy the conditions that

i. $\|v \oplus w\|_{m+n}=\max \left\{\|v\|_{m},\|w\|_{n}\right\}$

ii. $\|\alpha v \beta\|_{n} \leq\|\alpha\|\|v\|_{m}\|\beta\|$

for $v \in \mathbb{M}_{n}(V), w \in \mathbb{M}_{m}(V), \alpha \in \mathbb{M}_{n, m}(\mathbb{C})$ and $\beta \in \mathbb{M}_{m, n}(\mathbb{C})$, where $\oplus$ is defined to be

$$
v \oplus w=\left(\begin{array}{cc}
v & \mathbf{0} \\
\mathbf{0} & w
\end{array}\right)
$$

This gives us an abstract operator space.

Example 4.1.2. Let $V$ be a closed subspace of $\mathscr{B}(H)$, for some Hilbert space $H$. Then $\mathbb{M}_{n}(V) \subseteq \mathbb{M}_{n}(\mathscr{B}(H))$, where $\mathbb{M}_{n}(\mathscr{B}(H))$ can be viewed as $\mathscr{B}\left(H^{n}\right)$. As $H^{n}$ has a canonical norm as a Hilbert space, $\mathscr{B}\left(H^{n}\right)$ has a canonical norm also, thus we can define each $\|\cdot\|_{n}$ to be the inherited norm from $\mathscr{B}\left(H^{n}\right)$. This is called a concrete operator space.

A standard reference for the study of operator spaces is Effros and Ruan's textbook [3]. By Ruan's Theorem (See Theorem 2.3.5, [3]), every Banach space $V$ with an operator space structure upon it can be embedded into $\mathscr{B}(H)$, for some Hilbert space $H$, in such a way that the norm $\|\cdot\|_{n}$ of $\mathbb{M}_{n}(V)$ is identical to the inherited norm from $\mathscr{B}\left(H^{n}\right)$ as described in Example 4.1.2, so in fact all abstract operator spaces are concrete operator spaces.

Let $V, W$ be Banach spaces with operator space structures. For any operator $T: V \rightarrow W$, we can naturally define operators $T_{(n)}$ from $\mathbb{M}_{n}(V)$ to $\mathbb{M}_{n}(W)$ by applying $T$ to each entry in a matrix $v \in \mathbb{M}_{n}(V)$. That is,

$$
T_{(n)}\left(\begin{array}{ccc}
v_{1,1} & \cdots & v_{n, 1} \\
\vdots & \ddots & \vdots \\
v_{1, n} & \cdots & v_{n, n}
\end{array}\right)=\left(\begin{array}{ccc}
T\left(v_{1,1}\right) & \cdots & T\left(v_{n, 1}\right) \\
\vdots & \ddots & \vdots \\
T\left(v_{1, n}\right) & \cdots & T\left(v_{n, n}\right)
\end{array}\right)
$$

It follows easily from the axiom $\|v \oplus w\|_{m+n}=\max \left\{\|v\|_{m},\|w\|_{n}\right\}$ that the norms $\left\|T_{(n)}\right\|_{n}$ form a sequence

$$
\|T\|_{1} \leq\left\|T_{(2)}\right\|_{2} \leq \cdots \leq\left\|T_{(n)}\right\|_{n} \leq \cdots
$$

and although each member of this sequence is finite, as $n \rightarrow \infty$ it's possible for $\left\|T_{(n)}\right\|_{n} \rightarrow$ $\infty$ as well. We define the completely bounded norm to be

$$
\|T\|_{c b}=\sup \left\{\left\|T_{(n)}\right\|_{n}: n \in \mathbb{N}\right\}
$$


and say that an operator $T: V \rightarrow W$ is completely bounded if $\|T\|_{c b}<\infty$. In the case that $V=W$, the set of all complete bounded operators $T: V \rightarrow V$ form an algebra, which we call the algebra of completely bounded operators.

We apply this to $A(\Gamma)$ by utilising another fact shown in [3]; given an operator space structure on a Banach space $V$, there exists a canonical way to put an operator space structure of $V^{*}$, the Banach space dual of $V$. This is useful to us, as the dual of $A(\Gamma)$ is naturally contained in $\mathscr{B}\left(L^{2}(\Gamma)\right)$ (a result shown in [4], but the abelian case is illustrated in $\left.{ }^{1}\right)$. As $L^{2}(\Gamma)$ is a Hilbert space, $\mathscr{B}\left(L^{2}(\Gamma)\right)$ has a natural operator space structure on it, from which we obtain a canonical operator space structure on $A(\Gamma)$.

For any $\varphi \in M A(\Gamma)$, we have shown the construction of an operator $m_{\varphi}: A(\Gamma) \rightarrow A(\Gamma)$. As we have shown $A(\Gamma)$ is an operator space, it is natural to ask whether a given operator $m_{\varphi}$ is completely bounded. This allows us to define the completely bounded multiplier norm on $M A(\Gamma)$ to be

$$
\|\varphi\|_{c b}:=\left\|m_{\varphi}\right\|_{c b} \quad \varphi \in M A(\Gamma)
$$

Definition 4.1.3. The set of all $\varphi \in M A(\Gamma)$ with $\|\varphi\|_{c b}<\infty$ is called the completely bounded multipliers, and is denoted $M_{c b} A(\Gamma)$.

To confirm that $M_{c b} A(\Gamma)$ is indeed an algebra, consider $\varphi_{1}, \varphi_{2} \in M_{c b} A(\Gamma)$. Then

$$
\left\|\varphi_{1} \varphi_{2}\right\|_{c b}=\left\|m_{\varphi_{1} \varphi_{2}}\right\|_{c b} \leq\left\|m_{\varphi_{1}}\right\|_{c b}\left\|m_{\varphi_{2}}\right\|_{c b}=\left\|\varphi_{1}\right\|_{c b}\left\|\varphi_{2}\right\|_{c b}<\infty
$$

In general, we have $B(\Gamma) \subseteq M_{c b} A(\Gamma) \subseteq M A(\Gamma)$, with $\|\cdot\|_{m} \leq\|\cdot\|_{c b} \leq\|\cdot\|_{B(\Gamma)}$, but in the case that $\Gamma$ is an amenable locally compact group, $B(\Gamma)=M_{c b} A(\Gamma)$ isometrically. Thus an idempotent of small norm in $B(\Gamma)$ is an idempotent of small(er) norm in $M_{c b} A(\Gamma)$. These results are detailed in [12].

\subsection{Idempotent completely bounded multipliers}

Theorem 4.2.1. Let $\chi_{A} \in M_{c b} A(\Gamma)$ be the characteristic function for some non-empty $A \subseteq \Gamma$. Then the following are equivalent.

$$
\begin{aligned}
& \text { i } A \text { is an open coset in } \Gamma \\
& \text { ii }\left\|\chi_{A}\right\|_{c b}=1 \\
& \text { iii }\left\|\chi_{A}\right\|_{c b}<\frac{1+\sqrt{2}}{2}
\end{aligned}
$$

The equivalence of $\mathrm{i}$ and ii is shown in [6]. Surely ii $\Longrightarrow$ iii, hence it suffices to show that iii $\Longrightarrow \mathrm{i}$. In order to prove this, let us first observe the following.

\footnotetext{
${ }^{1}$ If $\Gamma$ is abelian, then $A(\Gamma)=L^{1}(G)$, which has dual space $L^{\infty}(G) . L^{\infty}(G)$ can easily be seen as a set of bounded linear operators on $L^{2}(G)$, which by Plancherel theorem, is isometric to $L^{2}(\Gamma)$
} 
Lemma 4.2.2. For any $s \in S$ and $t \in \Gamma$, if $s t \in S$ (resp. $t s \in S$ ), then $s t^{n} \in S$ for every $n \in \mathbb{N}$ (resp. $t^{n} s \in S$ for every $n \in \mathbb{N}$ ).

Proof. By translation, we may (and shall) suppose that $s=e$, the identity of $\Gamma$. Consider $\Gamma_{0}$ be the (abelian) group generated by $t$, then

$$
\left\|\chi_{S \cap \Gamma_{0}}\right\|=\left\|\chi_{S \cap \Gamma_{0}}\right\|_{c b} \leq\left\|\chi_{S}\right\|_{c b}<\frac{1+\sqrt{2}}{2} .
$$

So by the main theorem of [10] (or by the proof of Theorem 3.2.8, using Proposition 3.1.4), we see that $S \cap \Gamma_{0}=\Gamma_{0}$. This gives the lemma.

To prove 4.2.1, we want to compute the completely bounded multiplier norm of our given characteristic function. However, the definition of the completely bounded multiplier norm isn't very easy to work with. Luckily, there is an easier way to compute the norm. If we define $M_{\varphi}: \Gamma \times \Gamma \rightarrow \mathbb{C}$ to be the function $M_{\varphi}(s, t)=\varphi\left(s^{-1} t\right)$, then we can consider $M_{\varphi}$ to be $\left[M_{\varphi}(s, t)\right]$, a (possibly infinite) matrix, indexed by elements in $\Gamma$. From here, we can define

$$
\|\varphi\|_{\text {Schur }}:=\sup _{\|k\|=1}\left\|M_{\varphi} \bullet k\right\|
$$

where $k$ is taken from the collection of infinite matrices indexed by $\Gamma$ with finitely many nonzero entries, and $\bullet$ is Schur, or entrywise, multiplication, i.e.

$$
\left(M_{\varphi} \bullet k\right)(s, t):=M_{\varphi}(s, t) k(s, t)
$$

This gives us the Herz-Schur multiplier norm. As shown in [1], $M_{c b} A(\Gamma)$ is isometrically isomorphic to the space of Herz-Schur multipliers, so in fact, $\|\cdot\|_{S c h u r}=\|\cdot\|_{c b}$. Using this, we can prove the following.

Proposition 4.2.3. If $\varphi \in M_{c b} A(\Gamma)$ is an idempotent function such that

$$
F_{0}=\left(\begin{array}{lll}
1 & 1 & 1 \\
1 & 1 & 0 \\
1 & 0 & 1
\end{array}\right)
$$

is a submatrix of $M_{\varphi}$, then $\|\varphi\|_{c b} \geq \frac{1+\sqrt{2}}{2}$

Proof. Using the orthogonal matrix $U:=\frac{1}{2}\left(\begin{array}{ccc}0 & \sqrt{2} & \sqrt{2} \\ \sqrt{2} & 1 & -1 \\ \sqrt{2} & -1 & 1\end{array}\right)$ and the vector $\xi:=\frac{1}{2}\left(\begin{array}{c}\sqrt{2} \\ 1 \\ 1\end{array}\right)$, we see that

$$
\left\|F_{0}\right\|_{\text {Schur }} \geq\left\|F_{0} \bullet U\right\|_{\mathscr{B}\left(\ell^{2}\right)} \geq \frac{\left\|\left(F_{0} \bullet U\right) \xi\right\|_{\ell^{2}}}{\|\xi\|_{\ell^{2}}}=\frac{\sqrt{26}}{4}>\frac{1+\sqrt{2}}{2} .
$$


It is shown in [7] that $\left\|F_{0}\right\|_{c b}$ is actually equal to $\frac{9}{7}$, but we only need to know it is greater than $\frac{1+\sqrt{2}}{2}$ to prove Theorem 4.2 .1

Proof of Theorem 4.2.1] By [12, Corollary 6.3 (i)], we may (and shall) suppose that $\Gamma$ is discrete. Also, applying a translation if necessary, we suppose that $e \in S$. So it remains to prove that $S$ is a subgroup of $\Gamma$.

By Lemma 4.2.2, we see that if $u \in S$, then $u^{n} \in S$ for every $n \in \mathbb{N}$. Thus it remains to show that $S$ is closed under multiplication.

We next claim that if $u, v \in S$, then either $u v \in S$ or $v u \in S$. Indeed, assume towards a contradiction that both $u v \notin S$ and $v u \notin S$. Then the submatrix of $M_{\chi_{S}}$ with rows $e, u^{-1}, v^{-1}$ and columns $e, u, v$ is

$$
\left(\begin{array}{ccc}
\chi_{S}(e) & \chi_{S}(u) & \chi_{S}(v) \\
\chi_{S}(s) & \chi_{S}\left(u^{2}\right) & \chi_{S}(u v) \\
\chi_{S}(v) & \chi_{S}(v u) & \chi_{S}\left(v^{2}\right)
\end{array}\right)=\left(\begin{array}{ccc}
1 & 1 & 1 \\
1 & 1 & 0 \\
1 & 0 & 1
\end{array}\right)
$$

by the previous paragraph. This contradicts the previous discussion.

Finally, suppose that $u, v \in S$, the proof is completed if we can show that $u v \in S$. The claim shows that either $u v \in S$ or $v u \in S$. Assume the latter holds, then from Lemma 4.2.2 with $s=v$ and $t=u$, we obtain that $v u^{-1} \in S$. Since we must have $u^{-1} \in S$, this in turn implies, by a similar argument, that $v^{-1} u^{-1} \in S$. But then, since $v^{-1} u^{-1}=(u v)^{-1}$, we must have $u v \in S$. Hence, in any case, $u v \in S$, and the proof is completed. 


\section{Bibliography}

[1] M. BożEJKO, AND G. FENDLER, 'Herz-Schur multipliers and completely bounded multipliers of the Fourier algebra of a locally compact group', Boll. Un. Mat. Ital. A (6) 3 (1984) 297-302.

[2] D. Cohn, Measure Theory, (Springer Science + Business Media, New York).1980

[3] E.G. EFFros, AND Z.-J. RuAN, Operator spaces, (London Mathematical Society Monographs. New Series, 23, The Clarendon Press, Oxford University Press, New York). 2000

[4] Eymard, P., 'L'algbre de Fourier d'un groupe localement compact', Bulletin de la Socit Mathmatique de France 92 (1964) 181-236.

[5] G. B. Folland, A Course in Abstract Harmonic Analysis, (CRC Press, Inc.).1995

[6] M. Ilie, AND N. Spronk, 'Completely bounded homomorphisms of the Fourier algebras', J. Functional Analysis 225 (2005) 480-499.

[7] R. H. Levene, 'Norms of idempotent Schur multipliers', New York J. Math. 20 (2014) 325-352.

[8] J. Mudge, And H. Pham, 'Idempotents with Small Norm', J. of Funct. Anal. (2016) http://dx.doi.org/10.1016/j.jfa.2016.02.011.

[9] W. Rudin, Fourier analysis on groups, (Interscience Tracts in Pure and Applied Mathematics, No. 12, Interscience Publishers (a division of John Wiley and Sons), New York-London). 1962

[10] S. SAEKI, 'On norms of idempotent measures', Proc. Amer. Math. Soc. 19 (1968) 600-602.

[11] S. SAEKI, 'On norms of idempotent measures. II', Proc. Amer. Math. Soc. 19 (1968) 367-371.

[12] N. Spronk, 'Measurable Schur multipliers and completely bounded multipliers of the Fourier algebras', Proc. London Math. Soc. (3) 89 (2004) 161-192.

[13] A.-M. P. STAN, 'On idempotents of completely bounded multipliers of the Fourier algebra $A(G)$ ', Indiana Univ. Math. J. 58 (2009) 523-535. 